\title{
Pattern changes in step count accumulation and peak cadence due to a physical activity intervention
}

\section{Abstract}

Objectives: The purpose was to determine if a 12-week weight loss intervention with a physical activity (PA) component would lead to changes in steps/day, step count accumulation patterns, and peak cadence.

Design: Randomized controlled trial

Methods: Overall, 121 overweight/obese White and African-American adults (ages 35-64 yrs) were randomized to a diet education plus PA education and behavior change intervention group $(\mathrm{DE}+\mathrm{PA})$ or diet education and behavior change group $(\mathrm{DE})$. The $\mathrm{DE}+\mathrm{PA}$ intervention was designed to increase steps/day, and steps at moderate-to-vigorous intensity. The Actigraph GT3X+ accelerometer was used to measure steps accumulated in different cadence bands (119, 20-39, 40-59, 60-79, 80-99, 100-119, 120+ steps/min), and peak 1-min, 30-min and 60-min cadence. Pre- to post-intervention changes in steps/day, step count within each cadence band, and peak cadences were compared within groups using paired sample t-test and between groups after adjustment for baseline values of the same variable using ANCOVA.

Results: Ninety participants had valid data (44 in the DE+PA group). Change in steps/day was not significantly different between the groups. However, participants in the DE+PA group accumulated significantly more steps at post-intervention in the 80-99, 100-119, and 120+ cadence bands, all $p<0.02$. The DE+PA group increased step counts accumulated within the $100-119$ (463 \pm 1092 vs $56 \pm 546$ step counts; $p=0.01)$ and $120+(390 \pm 999$ vs $34 \pm 321$ step counts; $\mathrm{p}=0.03$ ) cadence bands, as well as peak 60 -min cadence when compared to the DE group. Conclusions: Non-significant changes in steps/day following a PA intervention may mask changes in steps accumulated at moderate-to-vigorous intensity cadences.

Key Words: peak cadence; physical activity guidelines; walking; activity counts 


\section{i. Introduction}

Physical activity is a cornerstone of the prevention of obesity and related chronic diseases. ${ }^{1}$ In order to prevent unhealthy weight gain it is suggested that adults engage in the equivalent of 300 minutes of moderate-intensity aerobic activity each week. ${ }^{1}$ Walking is such an activity and a device such as a pedometer can be used to motivate and monitor progress towards step-based goals. Interventions that have used pedometers as motivational tools have been effective in increasing total physical activity (PA) levels. ${ }^{2,3}$ Meta-analyses reveal that the expected average incremental increase over baseline PA is approximately $2000-2500$ steps/day. ${ }^{2,3}$ However, little is known about how individuals implement these changes (e.g., what patterns of change are evident and at what intensity these changes occur) and whether any increases in steps are at moderate intensity; an intensity which is recommended in guidelines. ${ }^{1}$ Most pedometers only output total steps accumulated during the day but this limitation can be overcome by the use of accelerometers. Accelerometers are capable of outputting total steps per day and can also be used to estimate cadence (steps/min) from timestamped data collected at resolutions of one or more data points per minute. This time-stamped feature can be used to examine, in detail, patterns of how individuals and groups accumulate (or reduce) step counts during the day. Time-stamped step accumulation patterns have been categorized generally into incrementally-grouped cadence bands for both adults ${ }^{4}$ and youth. ${ }^{5}$ Descriptive analyses using these categories have revealed that a relatively small portion of daily steps are accumulated at higher ( $\geq 100 \mathrm{steps} / \mathrm{min})$ cadences, considered a threshold for moderate intensity physical activity (MVPA) in adults. ${ }^{6}$ It is important to accumulate steps at cadences $\geq 100$ steps/min because MVPA is the recommended intensity for health-enhancing PA. ${ }^{1}$ The purpose of this analysis was to determine if a 12-week randomized controlled trial with

a PA intervention group would lead to changes in overall steps/day and step count accumulation patterns within and between groups. 


\section{ii. Methods}

Written informed consent was provided by all participants and the research protocols were approved by the Pennington Biomedical Research Center's Institutional Review Board, approval number \#PBRC-11028. The funding agency had no influence on the design, data collection, data analysis or required approval for publication. We performed secondary analyses of accelerometry data collected as part of a previously published randomized controlled trial conducted among overweight and obese white and Africa-American adults from the Lower Mississippi Delta (LMD) region of the United States. ${ }^{7}$ The intervention primary outcome was change in weight while increases in MVPA were among the secondary outcomes. The study methods pertinent to this analysis are summarized here. Briefly, the trial was a randomized 12week comparative evaluation of diet education and behavior change (DE) and diet education plus a pedometer-based PA intervention (DE+PA). Accelerometry was implemented as an outcome measure (pre- and post-intervention).

As stated in the primary outcomes paper, ${ }^{7} 22$ participants were excluded from the main analysis due to drop-out and loss to follow-up. This made 99 participants available for the primary outcome analysis and 90 of these had full and valid accelerometer data (defined below). Thus, the current analysis was limited to 90 overweight and obese White and African American adults (age 35-64). Participants in the DE arm attended group education and behavior change sessions with an interventionist once a week for 4 weeks on how to prevent weight gain through changes in diet behavior only. They also received telephone calls fortnightly for the remainder of the intervention (8 weeks). In addition to the same diet education component and telephone calls, participants in the DE+PA arm received an Omron $\mathrm{HJ}-151$ pedometer (Omron Healthcare, Kyoko City, Japan) and additional education and behavior change strategies to increase daily step counts to a steps/day goal of $8,300-9,100$ which was to related to 30 min/day of MVPA in the LMD population . ${ }^{7}$ 
At both pre- and post-intervention, steps/day and cadence-based step count accumulation patterns were assessed using the Actigraph GT3X+ accelerometer (ActiGraph, Pensacola, FL). The GT3X+ has been shown to measure steps reliably ${ }^{8}$ and accurately at speeds over $54 \mathrm{~m} / \mathrm{min}$, however it still counts some but not all steps at slower speeds. ${ }^{9,10}$ The accelerometer was worn on an elastic belt positioned over the right hip, in line with the midaxillary line. Participants were instructed to wear the accelerometer for 7 consecutive days during waking hours and to remove the device for water-based activities (e.g., bathing, swimming). Data were collected at a sampling frequency of $80 \mathrm{~Hz}$ and later integrated to $60 \mathrm{sec}$ epochs to match the epoch used in previous research. ${ }^{4,5,11}$ The data were processed using the manufacturer's default processing filter. ActiLife software (version 5.6.4 or later; ActiGraph, Pensacola, FL) was used for data download and processing.

Daily wear time (hours and minutes) was computed using SAS code provided by the National Cancer Institute (NCl) at http://riskfactor.cancer.gov/tools/nhanes_pam/. A valid day was defined as having $\geq 10$ hours of wear and $\geq 4$ valid days ${ }^{12}$ were required for a participant to be included in the present analysis.

We quantified daily time (minutes) and steps accumulated in the following cadence bands: 0 (non-movement), 1-19 (incidental movement), 20-39 (sporadic movement), 40-59 (purposeful steps), 60-79 (slow walking), 80-99 (medium walking), 100-119 (brisk walking), and $120+$ steps/min (fast locomotor movements). ${ }^{4}$ Values for each cadence-related variable were calculated for pre- and post-intervention assessments. Additionally, peak 1-, 30-, and 60min cadence were calculated. ${ }^{4,5}$ These peak cadence indicators are respectively calculated by averaging the highest cadences observed in 1, 30, or 60 (not necessarily consecutive) minutes throughout the day, and subsequently averaging the values across days..$^{4,5}$ 
Descriptive statistics were computed for steps/day, time and steps accumulated within each incremental cadence band and the three peak cadence indicators. Within-group differences between pre- and post-intervention assessments were evaluated using paired samples t-tests. Between-group differences at pre-intervention were tested using One-Way ANOVA. Between-group differences from pre- to post-intervention in change in time and steps accumulated at the different incremental cadences, as well as the peak cadence indicators, were tested using ANCOVA controlling for pre-intervention values of the same variable tested. In addition, analysis was run using BMI as a covariate, but BMI was only significant for the change in mean time and steps accumulated at 120+ steps/min. Graphical methods were used to evaluate residual normality (qq-plots and histograms) and ensure model adequacy. Dependent variables which produced right-skewed residuals were transformed using the BoxCox method (with a positive shift equal to the minimum value of the dependent variable +1 ) to yield residual distributions that more closely approximated normality. Analyses were then conducted separately using the raw and transformed data. Because conclusions regarding statistical significance were identical in all but 1 instance (change in mean time and steps accumulated at $120+$ steps/min), we present results herein using the raw data for ease of interpretation. All analyses were performed using IBM SPSS Statistics (version 20.0) and statistical significance was defined as $p \leq 0.05$.

\section{iii. Results}

Pre- and post-intervention values for the 12-week study are presented in Tables 1 and 2. There were no significant mean differences at pre-intervention between groups in any of the steps variables. Figure 1 shows the overall pattern of changes in step counts accumulated in different cadence bands from pre- to post-intervention for both the DE+PA and DE groups. In the $\mathrm{DE}+\mathrm{PA}$ group there were significant mean increases from pre- to post-intervention for steps/day $(p=0.01)$ as well as mean increases in time and step counts accumulated within the 
80-99, 100-119, and 120+ steps/min cadence bands (all $p<0.02$ ). In addition, a significant decrease in time and step counts accumulated within the 1-19 cadence band $(p=0.03)$ was observed among the DE+PA group (see Table 1). There were significant pre- to postintervention changes in all peak cadence indicators for the DE+PA group (all $p<0.01$ ), see Table 2. In the DE group, no significant increases between pre- and post- intervention values for any step or cadence indicator were observed. The DE+PA group experienced significant increases in mean time and step counts accumulated within the 100-119 and $120+$ steps/min (see Figure 1) cadence bands when compared to the DE group ( $p=0.01$ and 0.03 , respectively). There were no differences in the mean time and steps accumulated at $120+$ steps/min when the data were transformed or when BMI was used as a covariate. Additionally, the change in peak 30- and 60-min cadence differed significantly between groups $(p=0.02$ and 0.01 , respectively, see Table 2 ). No other significant differences in mean change in step counts or time spent at different cadence bands, and peak cadence between groups were observed.

\section{iv. Discussion}

The purpose of this study was to describe step count accumulation patterns underlying intervention-elicited changes in PA in two intervention groups. Although there were no differences in changes in steps/day between groups, the DE+PA increased steps/day from preto post-intervention and there was a significant increase in the time and step counts accumulated at cadence bands associated with purposeful walking (cadence bands $>80$ steps/min). ${ }^{4}$ The small change in steps/day for the DE+PA group $(+1,063 \pm 2,429)$ was less than the 2,500 steps/day increase which has been consistently achieved in previous pedometerbased interventions ${ }^{3}$ and somewhat unexpected. However, this could be due to the difference in step counts normally observed between the GT3X accelerometer and pedometers. ${ }^{13,14}$. In addition, the DE+PA group had a significant, however small decrease in step counts accumulated in the 1-19 cadence band. Combined with the other results, this points out to a 
displacement of the accumulation of steps at low cadence to a higher cadence. The DE+PA group also had a significant increase in step counts accumulated in the 100-119 and 120+ steps/min cadence bands when compared to the DE group.

This study used a novel approach to provide a more detailed examination of patterns of PA behavior change in response to intervention expressed in terms of steps/day, step counts categorized by incremental cadence categories, and peak cadence indicators. Cadence is associated with PA intensity and related energy expenditure ${ }^{6}$ and the analyses presented herein offer insights into the relative intensity of step-based changes due to intervention. Cadence has the potential to be less variable between different measurement instruments than accelerometer activity counts (which are brand specific) and estimated time spent at different PA intensities (commonly derived from activity counts). The potential for between-instrument agreement of cadence outputs has not been extensively studied, but some devices like the activPAL have been shown to be highly accurate at measuring cadence, ${ }^{15}$ however it uses a different epoch length which could lead to disagreement.

As is evident from this analysis, the identification of time and step count changes linked with specific cadence bands can lead to different approaches to measuring and reporting the success of interventions. For example, if intervention success had only been inferred from significant differences in the relative increase in steps/day between groups, DE+PA intervention could have been considered a failure. There was no significant difference in change in steps/day observed between the two groups. However, the more granular analysis of exactly how these steps were accumulated, and time accrued in the different cadence bands, illuminated the fact that group-specific changes did indeed occur. Specifically, positive changes (in both accumulated time and step counts) were apparent in the DE+PA group in the cadence bands associated with MVPA. ${ }^{6}$ Further, there was a decrease in time and step counts accumulated in the 1-19 cadence band. This approach represents a potentially important 
improvement in describing intervention-based changes in PA. Continued research in this area is needed to determine whether or not time and/or step count changes in different cadence bands have discrepant impacts on health outcomes, and what magnitudes of these changes are clinically meaningful. It is possible that not all statically significant changes are associated with improved health.

It is interesting to note that there were significant changes in all peak cadence indicators from pre- to post-intervention in the DE+PA group. However, peak 1-min cadence did not change significantly from the change in the DE group. Peak cadence indicators are a product of both step/min magnitudes and time spent at these cadence levels. It appears that peak cadence indicators summarized over shorter durations (i.e., peak 1-min) are most affected by the actual steps/min values practiced and have some natural day-to-day variability that potentially obscures underlying behavior change. In contrast, the peak 30- and 60-min cadence indicators are shaped in large part by a persistence of effort that may more aptly reflect volitional changes produced from targeted interventions. Although the intervention targeted increases in overall accumulation of MVPA over the whole day as well as accumulating this in 10 minute bouts, it may be that peak 30- and 60-min cadence are a more responsive index of PA intervention success than peak 1-min. This conclusion is speculative at this point and should be evaluated for consistency across different types of interventions.

The study presented herein was carefully conducted; however, it is not without limitations. This is a secondary analysis of an intervention and as such we did not perform power analysis a priori and some of the non-significant findings could be due to the lack of power. In addition, a large number of statistical tests were conducted and some of the statistical significance could have been identified by chance. Cadence was estimated using $60 \mathrm{sec}$ epochs and at the lower range it represents a step count accumulation pattern and not an actual continuously defined stepping rate. If shorter epoch lengths were used the estimates could be 
different. Dall et al. ${ }^{16}$ used short epochs and demonstrated that most stepping behavior occurs in short intervals of $76 \pm 6$ steps/min. However, the level of accuracy required for such precise measurements to detect every distinct stepping bout is much higher than current devices can achieve. The results presented here cannot be generalized to participants in interventions that achieve a larger change in steps/day which might lead to more pronounced and different changes in step count accumulation patterns.

\section{v. Conclusion}

This analysis of a randomized controlled trial described changes in steps/day, detailed patterns of accumulated time and step counts in incrementally ascending cadence bands, and peak cadences realized with PA intervention. We demonstrated that an intervention-driven increase in steps/day was achieved through accumulating more time and step counts at cadence bands associated with purposeful movement or walking. In addition, participants also increased their peak 30-min and 60-min cadence, representing volitional persistence of effort. Although gradual, this simple form of pattern analysis of intervention data was relatively straight forward and replicable. Similar analytical approaches can lead to more precise investigation of the relationship between PA and health outcomes; however additional studies are needed to determine the clinical relevance of these step and cadence-based pattern changes.

\section{vi. Practical Implications}

- A physical activity intervention can lead to changes in pattern of step counts accumulation.

- After the intervention, individuals in the PA+DE group accumulated more time and took more steps at higher cadence bands than individuals in the DE group.

- Individuals in the PA+DE intervention increased their peak 60-min cadence, indicating persistence of effort to increase physical activity. 
vii. Acknowledgment

This work was support by ARS/USDA Cooperative Agreement \#58-6251-8-038. P.T.K. is supported, in part, by the Marie Edana Corcoran Endowed Chair in Pediatric Obesity and Diabetes. D.M.H. is supported by the NIHR Leicester-Loughborough Diet, Lifestyle and Physical Activity Biomedical Research Unit. 
viii. References

1. U.S. Department of Health and Human Services. 2008 Physical Activity Guidelines for Americans: Be Active, Healthy, and Happy! Washington, D.C.2008.

2. Bravata DM, Smith-Spangler C, Sundaram V, et al. Using pedometers to increase physical activity and improve health: a systematic review. JAMA. 2007;298(19):22962304.

3. Kang M, Marshall SJ, Barreira TV, et al. Effect of pedometer-based physical activity interventions: A meta-analysis. Res Q Exerc Sport. 2009;80(3):648-655.

4. Tudor-Locke C, Camhi SM, Leonardi C, et al. Patterns of adult stepping cadence in the 2005-2006 NHANES. Prev Med. 2011;53(3):178-181.

5. Barreira TV, Katzmarzyk PT, Johnson WD, et al. Cadence patterns and peak cadence in US children and adolescents: NHANES, 2005-2006. Med Sci Sports Exerc. 2012;44(9):1721-1727.

6. Tudor-Locke C, Rowe DA. Using cadence to study free-living ambulatory behaviour. Sports Med. 2012;42(5):381-398.

7. Harrington DM, Champagne CM, Broyles ST, et al. Steps ahead: a randomized trial to reduce unhealthy weight gain in the Lower Mississippi Delta. Obesity (Silver Spring). 2014;22(5):E21-28.

8. Jarrett H, Fitzgerald L, Routen AC. Inter-instrument reliability of the Actigraph GT3X+ ambulatory activity monitor during free-living conditions in adults. J Phys Act Health. 2014.

9. Connolly CP, Coe DP, Kendrick JM, et al. Accuracy of physical activity monitors in pregnant women. Med Sci Sports Exerc. 2011;43(6):1100-1105.

10. Tudor-Locke C, Barreira TV, Schuna JM, Jr. Comparison of step outputs for waist and wrist accelerometer attachment sites. Med Sci Sports Exerc. 2014. 
11. Barreira TV, Katzmarzyk PT, Johnson WD, et al. Walking cadence and cardiovascular risk in children and adolescents: NHANES, 2005-2006. American Journal of Preventive Medicine. 2013;45(6):e27-e34.

12. Troiano RP, Berrigan D, Dodd KW, et al. Physical activity in the United States measured by accelerometer. Med Sci Sports Exerc. 2008;40(1):181-188.

13. Barreira TV, Tudor-Locke C, Champagne CM, et al. Comparison of GT3X accelerometer and YAMAX pedometer steps/day in a free-living sample of overweight and obese adults. J Phys Act Health. 2013;10(2):263-270.

14. Barreira TV, Brouillette RM, Foil HC, et al. Comparison of older adults' steps per day using NL-1000 pedometer and two GT3X+ accelerometer filters. J Aging Phys Act. $2013 ; 21(4): 402-416$.

15. Grant PM, Dall PM, Mitchell SL, et al. Activity-monitor accuracy in measuring step number and cadence in community-dwelling older adults. J Aging Phys Act. 2008;16(2):201-214.

16. Dall PM, McCrorie PR, Granat MH, et al. Step accumulation per minute epoch is not the same as cadence for free-living adults. Med Sci Sports Exerc. 2013;45(10):1995-2001. 


\section{x. Figure Legends}

Figure 1. Pre- to post-intervention changes $(\Delta)$ in step counts accumulated at difference cadence bands in the DE (diet education only) group and the DE+PA (diet and physical activity education) group.

Note: Values presented as mean \pm standard deviation; $a$ = significant difference in increase compared to other group; $b$ = significant difference in decrease compared to other group. Significance was set at $p<0.05$. 
Table 1. Minutes and steps/day accumulated at different cadence bands by participants in both intervention groups.

\begin{tabular}{|c|c|c|c|c|c|c|c|c|c|}
\hline \multirow[b]{2}{*}{ Group } & \multirow[b]{2}{*}{$\begin{array}{l}\text { Assessment } \\
\text { Time Point }\end{array}$} & \multirow[b]{2}{*}{$\begin{array}{l}0 \text { Steps } \\
\text { Non- } \\
\text { Movement }\end{array}$} & \multicolumn{7}{|c|}{ Cadence Bands (steps/min) } \\
\hline & & & $\begin{array}{c}1-19 \\
\text { Incidental } \\
\text { Movement }\end{array}$ & $\begin{array}{c}20-39 \\
\text { Sporadic } \\
\text { Movement }\end{array}$ & $\begin{array}{c}40-59 \\
\text { Purposeful } \\
\text { Movement }\end{array}$ & $\begin{array}{c}\text { 60-79 } \\
\text { Slow } \\
\text { Walking }\end{array}$ & $\begin{array}{c}\text { 80-99 } \\
\text { Medium } \\
\text { Walking }\end{array}$ & $\begin{array}{c}\text { 100-119 } \\
\text { Brisk } \\
\text { Walking }\end{array}$ & $\begin{array}{c}120+ \\
\text { Faster } \\
\text { Locomotion }\end{array}$ \\
\hline \multicolumn{10}{|l|}{$\begin{array}{l}\text { Time } \\
\text { (min/day) }\end{array}$} \\
\hline \multirow{2}{*}{$D E+P A$} & Pre & $436 \pm 91$ & $363 \pm 78$ & $65 \pm 27$ & $20 \pm 12$ & $8 \pm 6$ & $4 \pm 3$ & $3 \pm 4$ & $2 \pm 6$ \\
\hline & Post & $458 \pm 123$ & $345 \pm 75^{b}$ & $64 \pm 29$ & $22 \pm 13$ & $9 \pm 6$ & $5 \pm 5^{a}$ & $7 \pm 9^{a}$ & $5 \pm 11^{a}$ \\
\hline \multirow{2}{*}{ DE } & Pre & $426 \pm 102$ & $349 \pm 69$ & $62 \pm 23$ & $18 \pm 9$ & $7 \pm 4$ & $4 \pm 3$ & $3 \pm 4$ & $1 \pm 2$ \\
\hline & Post & $415 \pm 88$ & $347 \pm 75$ & $64 \pm 31$ & $20 \pm 12$ & $8 \pm 5$ & $5 \pm 4$ & $3 \pm 4$ & $1 \pm 2$ \\
\hline \multicolumn{10}{|l|}{ Steps/day } \\
\hline \multirow{2}{*}{$\mathrm{DE}+\mathrm{PA}$} & Pre & - & $2,162 \pm 585$ & $1,787 \pm 764$ & $945 \pm 586$ & $519 \pm 319$ & $331 \pm 269$ & $347 \pm 501$ & $240 \pm 800$ \\
\hline & Post & - & $2,032 \pm 551^{b}$ & $1,772 \pm 833$ & $1,058 \pm 630$ & $619 \pm 416$ & $473 \pm 442^{\mathrm{a}}$ & $811 \pm 1018^{a}$ & $629 \pm 1361^{a}$ \\
\hline \multirow{2}{*}{ DE } & Pre & - & $1,990 \pm 480$ & $1,701 \pm 642$ & $884 \pm 447$ & $488 \pm 267$ & $339 \pm 280$ & $316 \pm 465$ & $89 \pm 266$ \\
\hline & Post & - & $2,014 \pm 605$ & $1,767 \pm 890$ & $934 \pm 592$ & $532 \pm 356$ & $416 \pm 335$ & $371 \pm 454$ & $123 \pm 262$ \\
\hline
\end{tabular}

Note: Values presented as mean \pm standard deviation; $a=$ significant increase from pre-intervention; $b=$ significant

decrease from pre-intervention; Significance was set at $p<0.05$. 
Table 2. Steps/day and peak cadence indicators for participants in both intervention groups.

\begin{tabular}{cccccc}
\hline \multirow{2}{*}{ Group } & & & \multicolumn{3}{c}{ Peak Cadence Indicator } \\
\cline { 5 - 6 } & Assessment & & & & \\
& Time Point & Total steps/day & Peak 1-min & Peak 30-min & Peak 60-min \\
\hline \multirow{2}{*}{ DE+PA } & Pre & $6,331 \pm 2,544$ & $95 \pm 16$ & $61 \pm 17$ & $48 \pm 16$ \\
& Post & $7,394 \pm 3,365^{\mathrm{a}}$ & $103 \pm 16^{\mathrm{a}}$ & $72 \pm 23^{\mathrm{a}, \mathrm{b}}$ & $59 \pm 23^{\mathrm{a}, \mathrm{b}}$ \\
\hline \multirow{2}{*}{ DE } & Pre & $5,807 \pm 1,820$ & $94 \pm 12$ & $59 \pm 13$ & $46 \pm 12$ \\
& Post & $6,160 \pm 2,573$ & $99 \pm 14$ & $62 \pm 16$ & $49 \pm 13$ \\
\hline
\end{tabular}

Note: Values presented as mean \pm standard deviation; $a=$ significant increase from

pre-intervention; $b=$ significant increase compared to $D E$; Significance was set at $p<0.05$. 



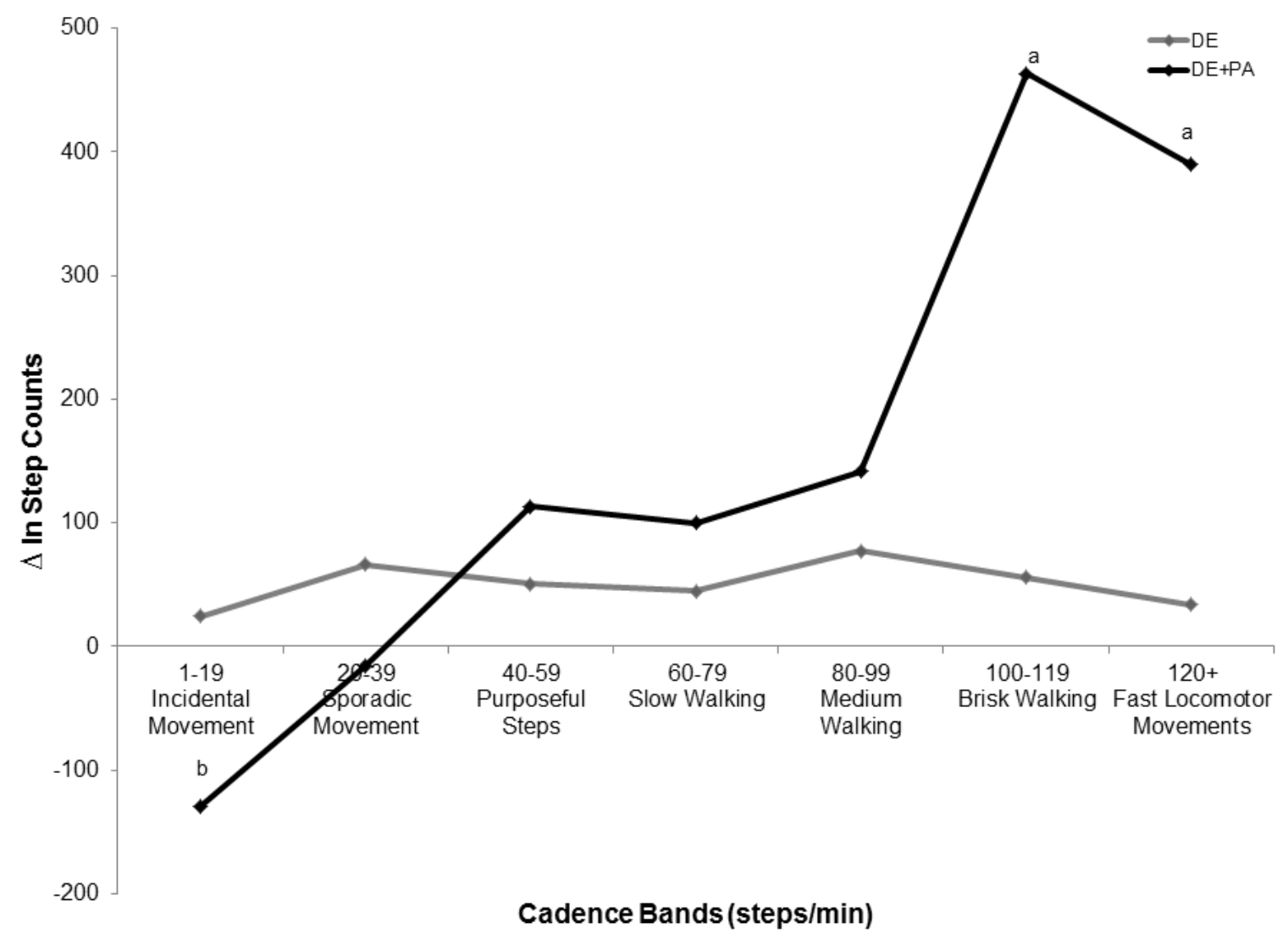

